Surgeon-Major Kleine, and in spite of repeated microscopical examinations no trace of trypanosome could ever be discovered. But the dog experiment in this case, as well as in that of the Togo mare, proved the existence of trypanosomes. This artificially-immunized animal, in spite of apparent good health, retained the trypanosome for years. It was in the same condition as the large antelopes and buffaloes which are, according to those most experienced in the tsetse regions, the principal source of tsetse infection. If we were to attempt to make whole herds of cattle immune in this way we would produce more permanent sources of infection, and teetse disease, which we hoped to exterminate by artificial immunization, would in this way be permanently retained. Under these circumstances it will be clearly understood that I do not think it advisable to combat tsetse disease by artificial immunization.

\section{Prophylactic Measures.}

We must look for other means of attacking this epidemic, and these are, fortunately, not wanting. Action might be taken either against the intermediary, the stinging fly, or the parasites themselves. Whether anything can be done against the Stomoxys and Tabanus species, which it is alleged carry the infection in India, the Philippines, Java, Mauritius, and South America, cannot at present be said, for too little is known of their habits, mode of life, and conditions of existence to render it possible to frame measures for their extermination. But it seems possible to do something against the African stinging flies, the Glossinae. It has often been observed that with the disappearance of large game tsetse flies and tsetse disease disappear too. Whether the disappearance is due to the drying up of the fountain of infection, the big game, must remain doubtful. But in every instance the cessation of tsetse disease in all places where wild game has been shot out or removed is an indisputable fact of which use should be made in the districts where tsetse disease and big game exist together. We must be clear on this point-which is the more profitable, a good stock of game or a good stock of cattle. Both cannot live together on African soil.

The measures taken against trypanosomes seem to work very successfully. With this ebject all animals which harbour trypanosomes must be sought out, and the danger removed by their extermination or slaughter, or, if kept to be slaughtered, they should be so isolated that they may no longer infect other animals. To ensure that all animals infected with trypanosome are included, not only must those actually ill be examined, but all those still apparently well but suspected.

The example of Java and Mauritius shows how useful is the diligent carrying out of such important measures, and how by neglecting them much harm may arise. In Mauritius, surra, which had been introduced from India by a cattle transport, was not known until recently, and, when recognized, no rational measures were at first taken. The result was that in two years all the horses and mules and most of the cattle died. In Java, on the other hand, the epidemic was quickly diagnosed and immediately taken in hand. The stalls were examined, diseased animals slaughtered, and those suspected efficiently isolated by sending them either to places where there were no stinging flies or to a neighbourhood where no cattle were. Thus the epidernic, which was quickly taken in hand, was checked, and the mortality kept within narrow bounds. By these means and by the precautionary measures taken against every infectious disease, we must in the future fight against trypanosome diseases.

Unfortunately these measures cannot be applied to trypanosome disease in men. It would not, of course, be difficult to isolate adequately in hospitals those pronounced to be suffering from sleeping sickness. Little, however, would be gained by this, for it is not these sick persons but the thousands of apparently healthy people who are already afflinted with try panosome who are the cause of the infection in this sickness, and how would it be possible to isolate thousands of healthy people? If we had a drug which would destroy the trypanosome in the blood, as malaria parasites are destroyed by quinine, then we might arrange a battle against trypanosomiasis on the pattern of the attack on malaria which has led to such good results. At present no such drug is at our disposal, but there are certain indications that it will yet be found.

For some time the use of preparations of arsenic has been attended by a not wholly unsuccessful result. Then Ehrlich discovered a remedy in a drug he called trypanroth, which gave decidedly better results than arsenic. Laveran and the members of the Liverpool School of Tropical Medicine succeeded still better with a combination of trypanroth and arsenic. Further, Wendelstadt has found that malachite green has a destructive effect on the trypanosome in the blood of experimental animals. All these investigations, however. are still in the stage of trial by laboratory experiments, and it is not yet known how they will work out in practice. They show, however, that a keen effort is being made to find a remedy against trypanosomiasis, and we can only hope that one may soon be discovered.

At all events, it is important that the inquiries into trypanosomiasis in man, begun so successfully by the English expeditions under Bruce, Dutton, and others, should be furthered by every means, and that all nations interested in combating this epidemic should lend assistance. The German nation is one of these. In Kamerun and Togo the existence of the disease has already been proved, and sleeping sickness quickly spreading from Üganda has already reached the borders of German East African territory, has perhaps already got beyond them. It is high time, then, that something should be done, and we would express the earnest hope that Germany may soon follow England's example.

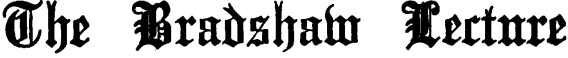

ON THE

\section{TREATMENT OF ENTERIC FEVER.}

\section{Delivered before the Royal College of Physicians of London on} November 15th, 1904 .

Bx F. FOORD CAIGER, M.D., F.R.C.P.LoND., Medical Superintendent, South-Western Fever Hospital, Stockwell, s.W.

\section{[ABSTRACT.]}

[AFTER some introductory remarks, in the course of which reference was made to the fact that the case-mortality of enteric fever remained to-day at a height of more than 15 per cent., and that in this country alone some 5,000 or 6,000 people annually died from the disease, Dr. Foord Caiger proceeded as follows:]

From a therapeutic point of view it may be stated broadly that the treatment of a case of enteric fever will probably be conducted on one of two lines: an active remedial method or a passive or so-called expectant method, each of which has its firm adherents.

Active or Remedial Methods of Treatment.

The methods of treating enteric fever by means of remedies which are assumed to be capable of exerting a direct controlling influence over the natural course of the disease may be appropriately referred to as either (1) specific, (2) antipyretic, or (3) antiseptic, according to their conception and to the nature of the agents employed, and they may conveniently be discussed under these headings.

Specific Treatment of Enteric Fever.

The remarkable success which has attended the treatment of diphtheria by the injection of antitoxic gerum not unnaturally encouraged the hope that a curative serum might be prepared which would prove equally efficacious in the case of typhoid fever. In the case of typhoid fever, however, the serum of a horse after repeated inoculations with the virus, though possessed of antibacterial properties, as in the case of diphtheria and tetanus, is found to be practically devoid of any antitoxic value, and consequently useless as a curative serum.

It should be mentioned, however, that Chantemesse claims to have produced a serum the nature of which is unknown, with which remarkable success in the treatment of enteric fever is stated to have been obtained.

[Reference was made to the protective method initiated by Professor A. E. Wright, consisting of the inoculation of sterile cultures of the typhoid bacillus which had been attenuated by exposure to heat. The Lecturer then continued :]

An investigation as to the value of Professor Wright's method of antityphoid inoculation was undertaken by the College of Physicians last year at the request of the War Office, and a special Committee of the College was appointed to inquire into it. Careful examination of the whole of the 
evidence available has tended to show that not only is a considerable degree of protection conferred by the inoculation, but that it is also capable of exercising a mitigating influence upon the severity of attack.

The Antipyretic Method of Treatment.

On the assumption that pyrexia is the most important factor in a case of enteric fever, the exhibition of remedies which have the power of reducing the body heat has been very extrnsively employed. An antipyretic effect may be brought about either by drugs, or the direct application of culd to the surface by means of cold baths, packs, sponging, etc., or by a combination of both. Of the various drugs which are recognized as possessing antipyretic power, the best for the purpose is undoubtedly quinine. Not nnly does quinine possess a marked antiseptic influence over living cultures of the typhoid bacillus, but its administration is not attended with the depressant influence on the heart. Moreover, it is believed not to exert any inhibitory influence on the elimination of toxins from the system, as is said to occur with drugs of the antipyrin class.

If quinine be given with the object of lowering the temperature, it must be administered in large doses- 15 to $20 \mathrm{gr}$. or more, preferably, twice in the twenty-four hours; or better still, according to the method recommended by Bouchard, which is to give four doses of $7 \frac{1}{2} \mathrm{gr}$., repeated at intervals of a quarter of an hour, in the evening of every third day during the first fortnight of the fever.

Fur my own part, I do not favour the ure of antipyretic drugs - at any rate, in antipyretic doses-in cases of moderate fever, as I firmly believe such pyrexia to be a natural element of defence against bacterial invasion. In cases, however, in which the pyrexia becomes excessive, and in itself represents an additional element of danger, by reason of its damaging effect on the cardiac muscle and the central nervous system, vigorous antipyretic measures are certainly indicated.

Of the various methods of treating enteric fever by means of cold applications to the surface, the most effective by far is the employment of the cold bath, advocated some forty years ago by Brand, of Stettin. General experience would show that the fatality from enteric fever can be brought down to somewhere about 7 per cent. by a thorough application of the cold bath treatment.

In this country, however, the use of the cold bath has been mainly restricted to the combating of hyperpyrexia in special cases. It is by no means improbable that it is to its salutary influence on the nutrition of the skin, and to its power of muintaining the excretory activity of both the skin and kidneys, that the cold bath mainly owes its marked superiority over all other therapeutic procedures, of which the primary aim is refrigeration.

The contraindications of the cold bath are well known. Its chief drawbacks are its cumbersomeness, and the increased tendency to relapse which is observed to follow its employment.

The "graduated bath," as it is termed, has been widely recommended. By this method the temperature of the bath, which at the moment of the patient's immersion is about $90^{\circ}$, is afterwards rapidly reduced by means of ice to $70^{\circ}$, or slightly lower. It is certainly more adapted to the condition of patients who are unable to bear the shock of sudden immer. sion at the lower temperature.

[Reference was made to the method advocated by Dr. James Barr, of Liverpool, for the treatment of typhoid fever by means of the continuous tepid bath, also to the methods of refrigeration by the ice-cradle, the ice-poultice, the wet pack, and cold sponging. Dr. Caiger then said:]

Of the various antipyretic drugs which have been used in combination with the cold bath sulphate of quinine is the only one which appears to merit any confidence.

The Antiseptic Method of Treatment.

Originally, no doubt, antiseptic drugs were administered on the supposition that they were competent to exert a direct bactericidal effect on the specific organism present in the intestine. It is now recognized that any attempt to achieve the destruction of bacilli in the lower region of the intestinal can al by the administration of antiseptic druss by the mouth is nothing short of futile, unless given in such strength or in such quantity as to be extremely prejudicial to the patient. But it is not unreasonahle to expect that, even when given in relatively small and harmless dcses, they might be capable of ex+rting some restricting influence on the multiplieation of bacteria in the mucous membrane and contents of the bowel, whether specific or otherwise. That such is actually $e$ ffected in respect to the various putre. factive organisms is obvious from the marked diminution in the fetor of the stools, which is usually soon apparent as the result of their administration. Further, recognizing, as we do, the extent to which the vital activities of micro.organisms are influenced by very slight changes in their environment, it is surely not too much to assume that as a result of the presence of even very minute quantities of an antiseptic, typhoid bacilli present in the intestinal mucosa, and at a somewhat later stage in the blood and tissues, may be so influenced as to be rendered in some degree less capable of elaborating their particular toxin than would be the case were the fluids in which they were living free from any trace of such substances.

Of the various drugs which are known to possess antiseptic properties, calomel is undoubtedly the one which has received the earliest and widest recognition. Murchison gave one or two doses of from 3 to $5 \mathrm{gr}$. during the first week of the attack, before there was much diarrhoea. The administration of calomel in this way has been widely practised, and as the result of my own experience of it, I am convinced that in suitable cases its effect is exceedingly beneficial. In exceptional instances, however, a dose of 3 or $4 \mathrm{gr}$. of calomel, ever when given not later than the end of the first week, may directly induce an intestinal irritation, as evidenced by diarrhoea and colic, which tends to persist and may seriously projudice the ultimate course of the attack. I have been so impressed with this that I have given up the routine use of calomel in the early stage of typhoid, and now restrict its administration to cases in which there exists some special indication for its employment. In the presence of severe headache, coupled with constipation and a very furred tongue, and in that class of attack in which diarrhoea of an offensive character and a tendency to abdomin 1 l distension are present at an early stage of the disease, especially when accompanied by high fever and undue restlessness, the marked improvement in the general condition of the patient which is brought about by the administration of calomel is frequently very striking. The value of the perchloride of mercury likewise, when given in frequent doses, has been much commended as an intestinal antiseptic. It is during the early stage of the attack up till about the middle of the second werk that the salts of mercury have usually been employed; and in respect to calomel, its use, except in very small doses, should be practically restricted to this period.

As the net result of my experience with the various antiseptic agents, I believe that some of them, when given in frequently-repeated doses, are capable of exercising a distinctly favourable influence on the course of the attack, even when their administration is not commenced until after the end of the first week. I do not, however, believe they are competent either to cut short the attack or to lessen to any appreciable degree the risk of either haemorrhage, perforation, or relapse, as has been contended by the most ardent advocates of the antiseptic method.

Drugs of the antiseptic class vary very much in their value, some of them, apparently, being next to useless, and the same drug is notnecessaril the most suitable in every case. I am of opinion, after a considerable experience of its use, that the administration of sulphurous acid, in 20 to 30 minim doses every two or three hours, is capable of checking fermentative changes in the bowel. A good plan is to give the sulphurous acid in an ounce of chloroform water, with the addition of 5 minims of syrup of lemon.

I am inclined to regard the oil of turpentine as a remery of somewhat greater value. It should be given in frequent doses from as early date as possible. It may be conveniently given in ro-minim cachets every two or three hours, or in the form of an emulsion with the addition of an equal quantity of spirits of chloroform and spirits of nitrous ether, as recommended by Sir J. IV. Mnore. The presence of marked albuminuria or of vesical catarrh, however, should preclude its emplos ment. In the latter case ro gr. of urotropine may with advantage he given three times daily, even though the urine be free from typhoid bacilli, but its influence in cystitis associated with the bacillus coli is very slight.

To one of these agents, in my opinion, a somewhat high value must be ascribed and that is the combination of quinine and nascent chlorine In its administration I have followed the formula advocated by Dr. Burney Yeo-that is, 40 minims of strong hydrochloric acid is pourea on to $30 \mathrm{gr}$. of powdered chlorate of potash in a r $2-0 \mathrm{z}$. bottle, which is filled up gradually with water, the mixture being frequently shaken as the water is being added, so as to absorb the gas as it is evolved. To the solution, when made, $24 \mathrm{gr}$ of sulphate of quinine :re added, and of this an ounce is given every two or three hours until convalescence is reached. Under this treatment the tendency to intestinal fermenta is reached. Under this treatment the tendency to intestinal fermentais usually well sustained, with corresponding benefit to the general aspect of the case.

During the last two years I have treated a series of cases with the 
essential oil of cinnamon. This agent was suggested to me by Mr. Carne Ross of Withington. The results, as far as they go, have been favourable, but the number of cases in which I have tried the drug is not yet sufficiently large to warrant a conclusion of very general application. Among 147 cases in which it was given, there was a mortality of 9.5 per cent.

The favourable effeșts which were noted as attending the administration of the drug were : the temperature in the majority of cases ran at a lower level than is customary in enteric fever; the patients remained for the most part drowsy throughout their illness, as a result of which mental rest was secured, and delirium was less frequent; intestinal decomposition, as evidenced by abdominal pain, distension, and fetor of the stools, was controlled to an extent which was really very striking, no single instance of meteorism occurring amongst the 147 cases which were treated with it.

It is well to give the drug in small doses to begin with, so as to accustom the patient to its pungent taste. By commencing with a dose of $2 \frac{1}{2}$ minims, and increasing it to 4 or 5 minims in the course of a fer days, the likelihood of romitiog being induced is materially diminished. Care should be taken that the quality of the drug is above reproach.

Being desirous of estimating the antiseptic influence which cinnamon oil is capable of exerting on the growth of the typhoid bacillus, one of my colleagues, Dr. A. F. Cameron, kindly undertook an investigation into the question. Working with a $x$ per cent. emulsion of cinnamon oil in distilled water coniaining the minimum necessary amount of mucilage, and a twenty-four hours' broth culture of the bacillus, which agglutinated readily with a $\mathrm{r}$ in 200 dilution of typhoid serum, he found that an appreciable, though slight, inhibitory influence on the growth of the typhoid bacillus begins to be exerted by cinnamon oil in a dilution of about $I$ in 2,600 , and that when its strength approaches $I$ in 1,000 its antiseptic effect is complete. Except in respect to the remarkable freedom from meteorism, the incidence under cinnamon of the more serious complications of typhoid fever presented nothing very striking, and in view of the fact that in three instances progressive cardiac enfeeblement developed where there was no special reason to anticipate its occurrence, I have recently adopted the practice of giving a grain of sulphate of quinine with each dose in all cases where a careful daily physical examination reveals a suspicion of circulatory failure; and the result has so far been reassuring.

Expectant or Symptomatic Treatment.

The treatment of enteric fever on "expectant" lines implies the recognition of our inability to exercise a direct control over the course of the disease. Having seen that the patient is properly nursed and suitably fed, we are content to adopt a waiting attitude, and while carefully watching for the appearance of any unfavourable symptoms, our efforts are confined to attempts at mitigating their severity, should any of them threaten to assume a dangerous proportion. But for my part I hold strongly to the belief that the adoption of an entirely expectant treatment is not only fallacious in its conception, but very mischievous in practice. When I am asked on what general lines I would recommend the treatment of a case of enteric fever, my answer is that, in the absence of a specific, I would treat the case on symptomatic lines, but that I would employ in addition, from the earliest date possible, such remedies of either an antipyretic or an antiseptic character, or both, as appeared to be specially suited to the character of the attack and the idiosyncrasy of the individual. I cannot help feeling, in view of the remarkable success which has been achieved with the cold-bath treatment abroad, that those who have charge of the treatment of enteric fever in hospitals are incurring some responsibility in withholding its use, save in the occasional instances where the cold bath is clearly inadmissible.

Treatment of Toxaemia.

We not infrequently encounter attacks in which toxaemia is very apparent-cases characterized by the occurrence of dark, foul-smelling evacuations, and fullness of the abdomen at quite an early stage of the attack, coupled with much nervous depression and a high temperature. In such cases antiseptics are especially indicated, and it is of first importance that their administration should be commenced as earl'y as possible. It is always well, as a preliminary measure, to rid the lower bowel of its putrid and offensive contents, and thus assist in bringing the intestinal tract into as sanitary a condition as possible. To achieve this much-to-be-desired result there is nothing so effective as calomel, and in this class of attack I would recommend the administration at the outset of 3 or $4 \mathrm{gr}$. of calomel in the case of an adult, followed in a couple of days by another dose of $3 \mathrm{gr}$. more.

It is, of cuure, desirable to get this part of the treatment over by the time when it is assumed that ulceration has become estahlished - that is to say, by the middle of the s+ cond week - but I would never hesitate in a case like this to give calomel at a somewhat later stage of the disease, if necessary. Should a free evacuation be not obtained, especi- ally if the abdomen still remains tumid, an enema of soap and water, with the addition of $10 z$. of turpentine, should be administered without delay. The antiseptic influence initiated by the calomel should be maintained by the regular administration every few hours of one or other of the antiseptic agents. In some cases, particularly where distension of the colon tends to persist, the continuous administration of turpentine in 10-minim doses will prove especially useful. Taking all things into consideration, I am disposed to regard the oil of cinnamon as the most suitable antiseptic to emplop in the large majority of such cases.

\section{Cases of Nervous Manifestation.}

There is another class of case in which the cinnamon treatment is especially serviceable, namely, that in which the patient evinces a constant tendency to anxiety and mental perturbation. Such patients, when fully under its influence, will often cease $\mathrm{fr}>\mathrm{m}$ worrying altogether, and pass the major portion of their time in quiet, restiul sleep. So important is the securing of mental rest to the victim of enteric fever, that in a good many cases it is no exaggeration to say that. treatment of the mind is the most cogent indication throughout. It is unfer these circumstances, too, that opium is so. valuable, and I would never hesitate to give opium until the clond had passed.

Other cases of enteric fever in which the brunt of the attack appears to fall upon the nervous system are often spoken of as ataxic: the most prominent features comprising generab prostration, muscular tremor, and early mental involvement, without, perhaps, there being any obvious symptoms of abdominal disturbance. For the treatment of these cases I know of nothing better, or so good, as the administration of quinine and chlorine every two or three hours. I have frequently been impressed with the steadying effect of this combination, even without the assistance of alcohol, of which, however, a small amount is usually called for under the circumstances.

\section{Treatment of Pyrexia.}

In enteric fever, in this country, at any rate, it is exceptional to meet with a temperature much exceeding $105^{\circ}$, though its occurrence is not by any means uncommon in the tropics. When, however, a patient's temperature approaches this level, the sy mptom pyrexia demands attention. It is especially in the later stage of the fever, when there is likely to be some degree of cardiac dilatation, that the continuance of a high degree of pyrexia is fraught with danger.

During the late period of the attack it is seldom wise to. put a person into a cold bath unless he have been acclimatized to it by previous experience, but in the early stage there would not be the same objection. As a means of reducing hyperpyr+xia in enteric fever I much prefer the cold pack to. the cold bath.

When given with this object the whole surface of the body from the neck downwards, with the exception of the arms and feet, should be enveloped in a couple of draw-sheets wrung out of cold, or even icecold, water. A mackintosh should previously be spread upon the bed, but there is no necessity to use a bed-cradle or further covering of any description. A blanket, however, may be thrown across the legs below the knees, and a hot-water bottle encased in flannel applied to the soles of the feet, a provision which will materially lessen the patient's discomfort. The pack shuuld be maintained for from fifteen to thirty minutes, its duration and the temperature of the water being regulated by the strength of the pul-e and the amount of shivering or cyanosis. which it induces. Some degree of shivering, however, must always bo expected. On removal of the pack the patient should immediately be wrapped in a warm, dry blanket, and as soon as the skin has obtained. a comfortable degree of warm th he should be placed between the sheets, and covered, preferably, with a sinele blanket. It is always desirable to give an ounce or so of brandy before the application of the pack, since, apart fru $m$ its primary effect in lessening shock and steadying the pulse, the antipyretio property of alcohol may be expected to supplement in some degree the action of the pack.

In occasional instances the reduction of temperature brought about by the bath or pack proves to be of a very temporary character, the pyrexia in the course of an hour or two attaining its former level. Under these circumstances the bath or park $k$ hould be repeated, and a dose of from 15 to $20 \mathrm{gr}$. of sulphate of quinine, combined with from 15 to 20 minims of laudanum, administered half an hour or so after the operation is completed. By the administration of quinine it is usually possible to prolong the effect of mechanical refrigeration-a fact well known to Brand and his followers.

The emplnyment of large doses of the synthetic antipyretics. is not to be recommended. Cold sponging of the surface though capable of reducing pyrexia when properly carried out, 
is chiefly valuable on account of its sedative influence on the nervous system. On account of its cooling and hypnotic effect, it is well to have the patient sponged down every morning with cold or tepid water, to which a few drops of eau-de-Cologne or spirits of lavender have been added, as a routine procedure, and to continue the practice until the establishment of defervescence. To be of any use, the sponge should be charged as full as it will hold, and the water effectively sopped or "soused" on to the skin.

\section{Treatment of Insomnia.}

In cases where the restlessness and sleeplessness fail to give way to cold sponging, a trial may be made of one of the numerous hypnotic drugs. In ordinary circumstances I prefer to give 30 to $40 \mathrm{gr}$. of chloralamide, or half that weight of trional, to be repeated in lesser amount at the expiration of a couple of hours, if necessary. In cases where insomnia exists in association with diarrhoea and abdominal pain, the preparations of opium are especially useful, and the administration of 15 to 20 drops of laudanum, or $10 \mathrm{gr}$. of Dover's powder, are either of them likely to prove a successful hypnotic. Retention of urine and a condition of rectal discomfort, the result of an inefficient action of the bowels, are sometimes directly responsible for a sleepless night.

Treatment of Abdominal Symptoms.

If diarrhoea be moderate, and unattended with abdominal pain, it may well be left alone, provided always that the dietary be suitable and its quantity not excessive. It has been well and truly said that careful daily inspection of the stools supplies the key, alike for regulating the diet and for the treatment of diarrhoea, in a case of typhoid fever. If in any case the number of the stools exceed four or five in the course of the twenty-four hours, and the amount evacuated be considerable, I have no hesitation in saying that the diarrhoea should be controlled without delay, since the continued drain of fluid, and consequent deprivation of nutriment which it involves, may speedily prove a greater tax on the patient's strength than is consistent with ultimate recovery. If the feeding is not at fault, the means of checking diarrhoes which I would recommend be first employed is the administration of a starch and opium enema. A couple of ounces of thin starch gruel, slightly warmed, and with the addition of half a drachm of laudanum, should be given immediately after each loose stool. One or two injections will usually suffice. Should, however, the diarrhoea persist, an opiate may next be tried by the mouth, as chlorodyne or Dover's powder. Astringent mixtures containing bismuth, catechu, tannic acid, chalk and opium, are often beneficial. The mineral astringents, such as sulphuric acid, acetate of lead and sulphate of copper, I confess, I do not like, and have long since ceased to use them. The application of an icebag to the abdomen is a remedy of undoubted value, especially when the diarrhoea is associated with distinct abdominal tenderness, but personally I much prefer the use of a large wet compress.

In the treatment of enteric fever there are few more valuable measures than the continued appljcation of a cold compress to the abdomen, in cases where definite tenderness can be elicited on slight pressure over some part of the surface, whether diarrhoea be actually present or not. Such tenderness, in the absence of marked distension, would appear to denote the presence of local peritoneal irritation at the site of one or more of the affected Peyer's patches. For the relief of intermittent abdominal pain, which is usually of a more or less colicky nature, the preparations of opium are probably unsurpassed.

The condition which we are far more frequently called upon to treat than diarrhoea is its converse, constipation. My practice is to give a simple enema of soap and water, not exceeding a pint, in the morning of every third day, in cases where the bowels fail to act spontaneously. If the quantity of milk allowed in the dietary be not in excess of the patient's digestive powers, this interval is not too long; but the enema may with advantage be given every second day, in the event of the stools produced being large, or their character not entirely satisfactory. Meteorism, if present in any marked degree, must always be regarded as a serious condition. and its relief in every case should be attempted.

It is always well to ascertain, if possible, which part of the bowel is distended, since not only map we be able to form some estimate of its immediate danger to life, but an indication may sometimes be obtained which may not be without some value in its treatment.

If the diet be carefully regulated in relation to the patient's digestive power, meteorism is not likely to be a frequent symptom. This is particularly true if an efficient antiseptic treatment have been instituted from an early stage of the illness, and the value of the oil of cinnamon in this respect I have already referred to. But should distension arise in spite of every care, and there be reason to believe that the small intestine is mainly concerned in its production, the administration of opium in moderate doses and the application of an icebag to the abdominal wall are the means most likely to prove successful. In severe cases, where tympanites develops rapidly at an early stage of the attack a full dose of opium should be at once administered. In my experience the value of drugs of the antiseptic class for the relief of tympanites, when once it has arisen is almost nil. Where there is reason to believe the meteorism to be mainly due to flatulent distension of the colon, enemata of soap and water containing $\mathbf{I} \mathrm{oz}$. of turpentine are usually of the greatest value. Under these circumstances the icebag had better be withheld, and, opium, too, unless the pain continue. It is in these cases that the passage of a long tube through the sigmoid flexure may be expected to afford some relief, though the procedure is by no means always successful.

Treatment of Cardiac Failure.

That the pulse should shew some loss of strength as the fever progresses is only natural, and in the event of such loss being moderate, no special treatment is required; but should the weakness become more pronounced, as evidenced by undue rapidity in relation to the temperature, dicrotism, or especially if a tendency to irregularity be noted, either in force or rhythm, the cautious administration of stimulant is usually advisable.

The form of alcohol best suited for the purpose as a rule is either brandy or whisky, of which, however, the quality, as also the quantity, should be regulated with discretion. In some cases the feebleness of pulse is associated with marked ventricular dilatation in which case pulmonary congestion is very prone to arise and seriously prejudice the issue. The good effect of digitalis under these circumstances is usually apparent, and it may with advantage be combined with strychnine. A certain amount of benefit, no doubt, would be derived from the administration of alcohol alone, but its influence often appears to be but temporary, and for producing a lasting effect it cannot compare in value with either stryohnine, quinine, or digitalis.

I rank myself with those who hold that in most cases of enteric fever, not only is alcohol not required, but that its emplopment is occasionally distinctly harmful, even when given in quantities which would not be considered excessive. The administration of a few ounces of alcohol, for instance, will sometimes markedly increase the restlessness in the case of a delirious person whose pulse is gond and circulation well sustained, especially when the patient is young and unaccustomed to stimulants.

The special indications which, I think, legitimately demand the ise of alcohol in typhoid fever may be enumerated as follows: Constant delirium and sleeplessness associated with muscular tremor, feeble circulation, and a dry brown tongue; undue weakness of the pulse without other sign, as already described; cardiac dilatation, cyanosis, pulmonary congestion, and pneumonia; hyperpyrexia, excessive diarrhœa, and intestinal perforation. Alcohol, moreover, may be given with advantage in the case of elderly persons, and to patients who are taking badly, as also to those who are the victims of a persistent feeling of depression. In this latter instance the best form in which to administer the alcohol is a good champagne.

Treatment of Intestinal Haemorrhage.

The dictum generally credited to a late very distinguished physician that "haemorrhage from the bowels in typhoid fever must be allowed to cure itself" is as true to-day as when the words were spoken.

The importance of absolute repose, alike of body, bowel, and mind, is universally acknowlerged. For the attainment of physical and mental rest we must largely rely on the care and common sense of a competent nurse, and obedience on the part of the patient; whereas rest for the ulcered and bleeding bowel can be secured by the prompt administration of a full dose of opium. Everything calculated to stimulate the circulation should for the time, of course, be studiously avoided, and the amount of nourishment should be reduced to the lowest possible point consistent with maintenance of the physical powers.

The influence which is exerted by the complete deprivation of fluids in keeping down the blond pressure is almost equally important. Giving astringents by the mouth is obviously futile, and the use of physiological haemostatics, as ergot and hamamelis, is almost, if not entirely, worthless. Adrenalin chloride in frequent doses, however, I still 
employ in cases where the pulse tension is particularly low. Calcium chloride in 10-gr. doses every three hours is deserving of a trial, particularly in cases where the hremorrhage, though slight, appears to be continuous. The application of an icebag to the right iliac region is apparently of real benefit in persons whose abdominal wall is not unduly thick, Having seen that the patient receives a full dose of opium, and that an icebag is carefully applied to the abdomen, our treatment of intestinal haemorrhage may be summed up in the single word "precaution," and of the various measures we adopt with this in view, I believe the complete deprivation of fluids, excepting an occasional fragment of ice, to be the most important.

\section{Treatment of Perforation.}

Personally, I am in complete agreement with those who hold that a moribund condition of the patient should be the only contraindication to operation. I believe that under very exceptional circumstances a more or less localized peritonitis of perforative origin may be recovered from, but the occurrence is so exceedingly rare as to justify one in entirely ignoring its likelihood when called on to decide upon the treatment.

- In regard to the time most favourable for operation, " there is no time like the present.". 'The accumulated experience of the last few years has clearly shown that if surgical treatment be postponed until reaction has set in not only may the chance of a favourable issue have been allowed to slip, but the only opportunity for operation also. From this the value of early diagnosis in the attainment of the best results will be apparent.

- Of the various symptoms which are recognized as indicative of the onset of perforation, the vecurrence of sudden pain at some particular spot in the abdomen is properly regarded as the most suggestive, and if this is quickly followed by local tenderness and some degree of constitutional disturbance, the suggestion is greatly strengthened. Unfortunately, in something like 20 per cent. of cases, the access of definite pain is wanting, in which case perforation in its initial stage is far more likely to escape observation. In the absence of local pain, however, the presence of even slight muscular rigidity, which, from previous examination, is known to have newly arisen, must be regarded as an indication of the highest value; and its import is greatly strengthened in the event of any increase in the pulse rate being detectable at the same time.

To those who have seen much of enteric fever, the fact is well recognized that we may encounter cases of perforation in which every local symptom usually associated with the condition is conspicuous by its absence, the general aspect of the case in other respects being quite characteristic. Apart from the evidence which may usually be derived from a careful examination of the abdomen, I have come to regard the facies as, perhaps, the most valuable indication. To one who is familiar with the patient's usual appearance, a slight but distinct change in the facies may be apparent. When, in addition, there is present, or has recently been noted, some degree of shivering, the inference that perforation has occurred is very strong indeed.

As confirmatory evidence of perforation, obliteration of the liver dullness, and the presence of fluid in the peritoneum are, of course, unrivalled; but in neither case are they likely to be apparent until a fairly confident diagnosis has been attained trom other signs.

It is a golden rule in the treatment of typhoid fever to make a careful examination of the abdomen daily, for in proportion as our previous examination has been recent, so are we in a better position to appreciate the moment when any change in its condition may have occurred. Accuracy of diagnosis should always be aimed at, but in view of the greatly improved results which have been attained of late, it is better to recommend an unnecessary laparotomy than to allow a patient who has perforated to die without receiving the chance which operation affords.

\section{“TICK FEVER"}

PHILIP H. ROSS, M.R.C.S., L.R.C.P.Lond., D.P.H Camb.

Government Bacteriologist, Uganda. and

\author{
A. D. MILNE, \\ M.B., C.M.Aberd. \\ Medical Officer, \\ Uganda Protectorate.
}

THE part played by ticks in the transmission of varieties of piroplasma has been known for some time, and recently Marchaux and Salimbeni have shown that a tick conveys the spirillary disease of fowls. A second generation of ticks is not necessary, but a tick fed on an infected fowl has shown itself capable of transmitting the disease for five months.

The paper by Marchaux and Salimbeni reached me here while working with Dr. Hodges at the spirillum fever. It occurred to us then that possibly the so-called "tick fever" might be due to a spirillum. On the whole, we thought it improbable, not on clinical grounds but because it seemed unlikely that the spirillum should have escaped notice till now ; but by the time we had examined about a dozen cases of spirillum fever we found, as we have already pointed out, that the spirilla might be exceedingly rare in blood taken even at the height of the fever from cases with well-marked clinical symptoms. If fortunate, we might find a spirillum after only a few minutes' search, or we might have to opend several hours before finding a single organism ; in some of the cases in which search was made through the whole of a large blood film only two or three spirilla were found. With these results it seemed possible that the spirillum might have been overlooked in cases of tick fever.

When Dr. Hodges went on leave, Dr. Milne carne from Hoima, in the Unyoro district, to take his place. Dr. Milne brought with him slides of blood he had taken from several cases of fever in natives, who attributed their illness to the bite of ticks in the huts of two of the native camps. On hearing of the possibility of spirilla being the cause of the disease, he very kindly handed on his slides to me to be stained and examined. In all 8 cases $I$ found spirilla present, in I case fairly numerous (about one in thirty fields); in the other cases very few and far between.

All the cases were of men who attributed their illness to tick bites. They were taken by Dr. Milne as cases of tick fever, and would appear in statistics as cases of that disease. It seems, therefore, allowable to say that certain cases diagnosed as tick fever are due to a spirillum, and, in the light of Marchaux and Salimbeni's work, it seems probable that the disease is inoculated by the bite of infected ticks. It is possible that there may be other parasites conveyed to man by ticks, and so it is possible that there may be more than one form of tick fever.

The only description of tick fever available here is that in Manson's Tropical Diseases. Daniels examined the blood of a case in the second week, with negative results, as would be expected were the disease due to spirilla. All the accounts given describe vomiting as a prominent symptom; in the European and Indian patients with spirillum fever this was the most prominent symptom. In some of the native cases there was vomiting, in others there was not. So also some of the native spirillum patients had a relapse, others had not. In one case of a native there was no relapse so far as temperature was concerned, but at the time when a relapse was expected he complained of severe headache and a féw spirilla were found in his blood, although that day and the next there was no rise of temperature. In both the European patients headache seemed to be the last manifestation of the disease, one of them also having dysenteric stools.

In the case of a European at present suffering from an initial attack of spirillum fever, the first symptoms were vomiting and dysenteric stools as described by Daniels. But in the absence of any full description of tick fever it is impossible to do more than refer to features common to spirillum fever as seen here, and tick fever as described in Manson's work.

Dr. Milne, as he states below, had ticks collected from the camps where the patients said that they had been bitten, and sent them to the British Museum for identification. A further batch of ticks has now been collected from one of the camps, and experiments will be made to see if they will give any disease to monkeys on whom they are to be fed.

\section{Note By A. D. Milne.}

The following notes on tick fever are the results of observation on some 8 cases which presented themselves as such at the Hoima Dispensary. Owing to there being no hospital in which to place the patients and the length of time necessary to arrive at facts by interpretation of three different languages, the clinical notes are meagre in the extreme, ever the temperature charts representing a twomile walk every evening to reach patients in different camps; but such as they are, from the result of Mr. Ross's examination of the blood films, it seems worth while placing. them on record.

Through the kindness of Mr. Grant, Collector of Masinde, a neighbouring station, specimens of the tick were obtained, and were sent to the British Museum to be identified. These ticks, apparently, have a wide distribution in Africa, as the German Vice-Consul at Entebbe informs me that one of the hief caravan roads in German East Africa, south of Victoria Nyanza, has just been closed owing tol constant sickness 